\title{
The Periodic Repolarization Dynamics Index Identifies Changes in Ventricular Repolarization Oscillations Associated With Music-Induced Emotions
}

\author{
Giuliano Cerruto ${ }^{1}$, Luca Mainardi ${ }^{1}$, Stefan Koelsch ${ }^{2}$, Michele Orini ${ }^{3}$ \\ ${ }^{1}$ Politecnico di Milano, Milan, Italy \\ ${ }^{2}$ University in Bergen, Bergen, Norway \\ ${ }^{3}$ University College London, London, UK
}

\begin{abstract}
The effect of music on cardiovascular dynamics may be useful in a variety of clinical settings.

The aim of this study was to assess whether listening to music characterized by different emotional valence affected ventricular periodic repolarization dynamics (PRD), a recently-proposed non-invasive index of sympathetic ventricular modulation.

The 12 lead ECG was recorded in 71 healthy volunteers exposed to six $90 \mathrm{~s}$ excerpts of pleasant music and unpleasant acoustic stimuli as well as six 90 s intervals of silence. A 20 s interval was allowed between excerpts during which the participants were asked to evaluate the previous excerpt. A simulation study was carried out to assess the capability of the algorithm of tracking fast small changes in PRD.

The simulation study shows that the algorithm implemented in this study has a time-frequency resolution sufficient to capture the fast dynamics observed in this study. PRD were higher during listening to both pleasant and unpleasant music than during silence. There was a (weak) trend for the PRD to be higher during listening to pleasant than unpleasant music that may indicate the existence of a (weak) interaction between the valence of music-induced emotions and sympathetic ventricular response. The PRD significantly increased during the 20 s interval in between conditions, possibly reflecting a sympathetic response to the evaluation task and/or to the expectation of the following excerpt.
\end{abstract}

\section{Introduction}

The cardiovascular response to music depends on multiple factors, but in general it includes an increase in both heart rate and respiratory rate with respect to silence, with a further increase during listening to exciting as opposed to relaxing music $[1,2]$. There is evidence that music can reduce pain and anxiety and may therefore be useful in a variety of clinical settings. Music-induced emotions affect heart rate variability [2], a non-invasive marker of cardiac autonomic nervous modulation. However, the effect of music-induced emotions to the ventricular activity is unknown. Ventricular repolarization, which corresponds to the T-wave of the surface ECG, is an important parameters in arrhythmia mechanisms and is affected by the sympathetic outflow directed to the ventricles.

The periodic repolarization dynamics index is a recentlyproposed vector-cardiographic parameter associated with ventricular sympathetic activity [3]. It measures the amplitude of low-frequency oscillations in the angle of the repolarization vector and has been shown to track changes induced by autonomic tests known to provoke a sympathetic response and it predicts sudden cardiac death [3,4]. Lowfrequency oscillations have also been observed in the ventricular action potential duration of heart failure patients during arousal elicited by watching dramatic sequences from a psychological horror film [5]. A recent theoretical study has suggested that these oscillations may be due to the effect of sympathetic modulation on calcium and potassium ionic currents [6].

The aim of this study was to assess whether listening to music characterized by different emotional valence affects sympathetic-associated PRD.

\section{Population study}

Seventy-five young volunteers (age $24.5 \pm 3.2$ years, 36 female) participated in a study designed to characterize the effects of acoustic stimuli with different emotional valence. Details about the experimental protocol can be found in $[2,7]$. Briefly, six excerpts of pleasant joyful instrumental music and unpleasant acoustic stimuli, as well as six intervals of silence, were presented to the subjects in a randomized order. Each excerpts lasted about $90 \mathrm{~s}$. The unpleasant stimuli were electronically manipulated counterparts of six musical pieces. For any of these stimuli, a new soundfile was created in which the original excerpt was recorded simultaneously with two pitch-shifted ver- 

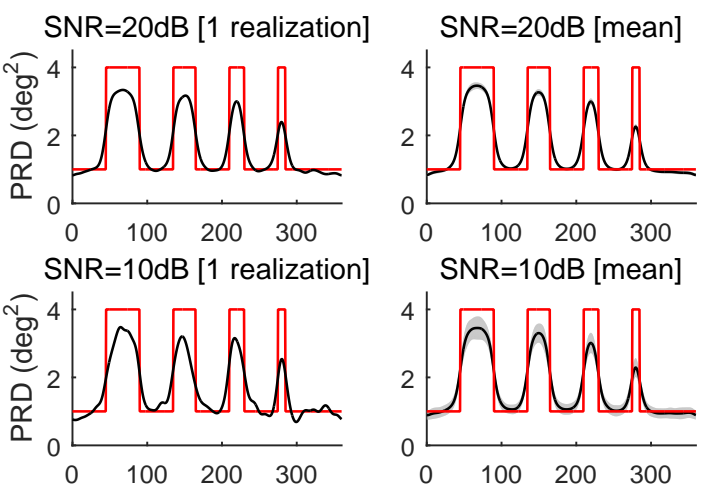

$\mathrm{SNR}=0 \mathrm{~dB}$ [1 realization]
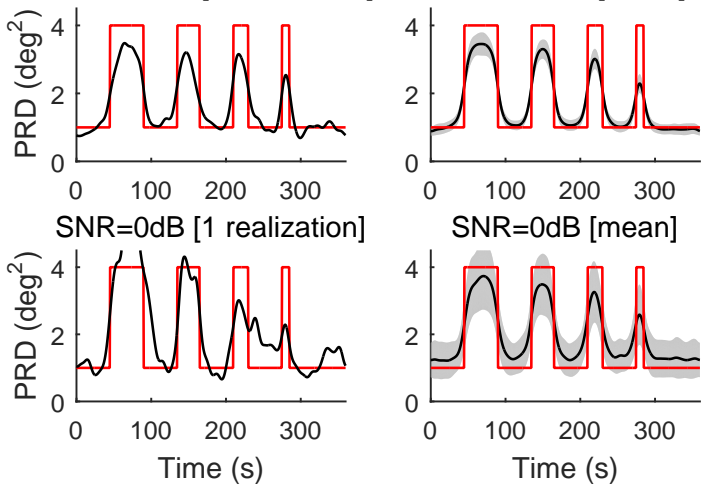

Figure 1. Simulation study. Estimation of sudden changes in sympathetic-associated PRD index. Left and right panels show results for 1 and 100 random model realizations, respectively. Continuous red line shows theoretical PRD, with first and last bursts lasting $45 \mathrm{~s}$ and $15 \mathrm{~s}$ respectively. On the right, black line represent the median trend and shaded area the interquartile range.

sions, and subsequently recorded backwards in order to introduce many dissonant structures. Then, series of Shepard tones were overlaid in order to match the metre of the original pleasant excerpts. The time interval between tones was chosen to match the tempo of the pleasant excerpts. All stimuli were matched for volume. At the end of each condition, about $20 \mathrm{~s}$ were allowed to indicate how pleasant or unpleasant the participants felt during it by using response buttons.

\section{Method}

Standard 12-lead ECG were measured using a 32 MREFA amplifier and digitized with a sampling rate of 1 KHz. The Dower's inverse transform was used to derive X, $\mathrm{Y}$ and $\mathrm{Z}$ orthogonal leads. ECG parameters were measured using Matlab algorithms developed on the course of other studies [8,9]. As described in [3], polar coordinates were derived and the elevation, $\phi$, was defined as the angle between the repolarization vector and the Y-axis, with $\phi=0$ deg defined as the vector pointing to the caudal direction. The azimuth, $\theta$, was defined as the angle between the vector on the transverse plane and the $\mathrm{X}$-axis. The angles were then weighted as:

$$
\tilde{\phi}(t)=\frac{\sum_{t} \phi(t) r(t)}{\sum_{t} r(t)} \quad \tilde{\theta}(t)=\frac{\sum_{t} \theta(t) r(t)}{\sum_{t} r(t)}
$$

where $r(t)$ represents the magnitude of the repolarization vector. It can be shown that the difference between repolarization vectors of consecutive beats $(i-1)$ and $i$ is [3]:

$$
\begin{aligned}
& d T_{i}=\arccos \left[\sin \left(\tilde{\phi}_{i-1}\right) \cos \left(\tilde{\phi}_{i-1}\right) \sin \left(\tilde{\phi}_{i}\right) \cos \left(\tilde{\phi}_{i}\right)+(2)\right. \\
& \left.\cos \left(\tilde{\phi}_{i-1}\right) \cos \left(\tilde{\phi}_{i}\right)+\sin \left(\tilde{\phi}_{i-1}\right) \sin \left(\tilde{\phi}_{i-1}\right) \sin \left(\tilde{\phi}_{i}\right) \sin \left(\tilde{\phi}_{i}\right)\right]
\end{aligned}
$$

The angle $d T_{i}$ is considered an estimate of the instantaneous degree of repolarization instability.

Finally, the PRD index was measured as the instantaneous power of $d T_{i}$ within the low-frequency spectral band $(0.04-0.15 \mathrm{~Hz})$, derived by integration of the timefrequency distribution of the signal obtained by evenly sampling $d T_{i}$ at $2 \mathrm{~Hz}$, i.e. $d T(t)$. Time-frequency representations were calculated using Cohen's class distributions as in previous studies $[10,11]$. Their temporal and spectral resolution of $11 \mathrm{~s}$ and $0.03 \mathrm{~Hz}$, respectively.

\subsection{Simulation study}

A simple simulation study was conducted to assess the ability of this technique to resolve rapid changes in ventricular dynamics in noisy conditions. The angle between repolarization vectors was considered to be an amplitude modulated complex exponential with central frequency equal to $f_{0}=0.1 \mathrm{~Hz}$. Furthermore, white Gaussian noise, $\xi(t)$, corresponding to SNRs equal to 20,10 and $0 \mathrm{~dB}$, representing good, average and bad signal quality was added:

$$
d T(t)=A(t) \exp \left(j 2 \pi f_{0} t\right)+\xi(t)
$$

The amplitude modulation was used to simulate sudden small bursts in sympathetic activity, each one of a duration between $10 \mathrm{~s}$ and $45 \mathrm{~s}$. These were assumed to correspond to squared-waves in the temporal evolution of the PRD (see Fig 1).

\subsection{Statistical analysis}

The distribution of data is illustrated using error bars showing the median values and the inter-quartile range. For each participant, the time-course of the PRD during pleasant, unpleasant and resting conditions was obtained by averaging PRD among the six intervals during which each one of these conditions was presented to the participant. Each PRD value was corrected by subtracting the baseline PRD value measured during the $20 \mathrm{~s}$ period preceding each excerpts, which corresponds to the interval during which the participants executed the self-evaluation task and waited for the following excerpts. Pair-wise comparisons were conducted using the paired non-parametric Wilcoxon signed-rank test. 

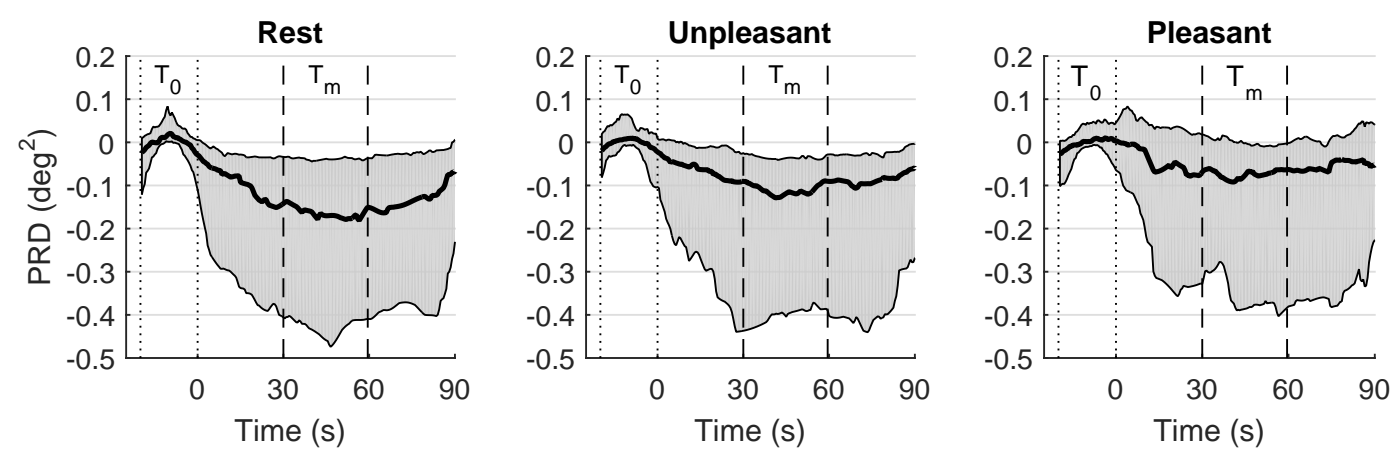

Figure 2. Time-course of baseline-corrected PRD during the three studied conditions. Solid line and shaded areas represent median and interquartile range among participants, respectively.
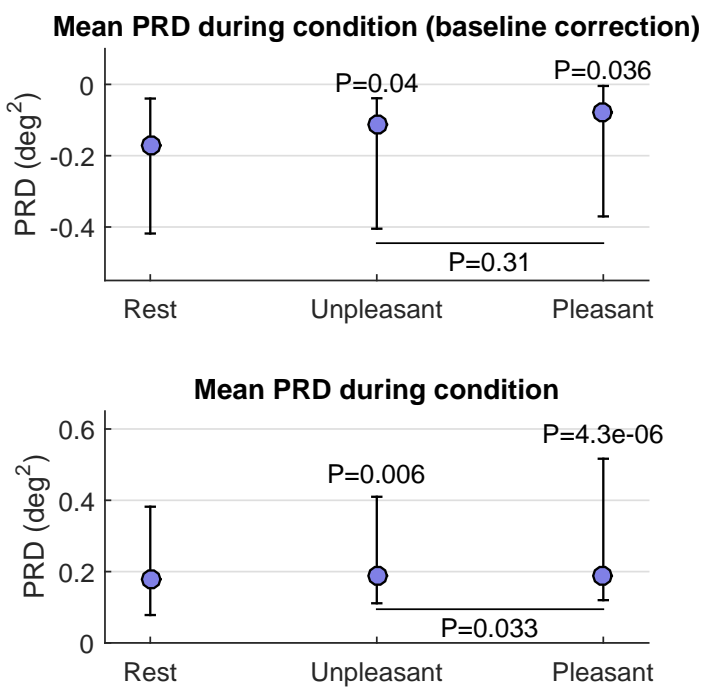

Figure 3. Mean PRD during resting, pleasant and unpleasant conditions, with (top) and without (bottom) baseline correction. Mean PRD was computed within an interval going from $30 \mathrm{~s}$ to $60 \mathrm{~s}$ after the onset of the condition (see interval $T_{\mathrm{m}}$ in Fig. 2). P-values reported on top of the errorbars are calculated with respect to the resting condition.

\section{4. $\quad$ Results}

\subsection{Simulation study}

The time-frequency analysis used in this study was able to detect small changes in the PRD even when their duration was as low as $10 \mathrm{~s}$ (Fig. 1). The Pearson's correlation coefficient between the theoretical PRD value (bold red line) and the estimated PRD during each one of the 100 model iterations (black line, left panels) were equal to $0.92 \pm 0.003$ (for $\mathrm{SNR}=20 \mathrm{~dB}$ ), $0.90 \pm 0.011$ (for $\mathrm{SNR}=10 \mathrm{~dB}), 0.75 \pm 0.065$ (for $\mathrm{SNR}=0 \mathrm{~dB})$.

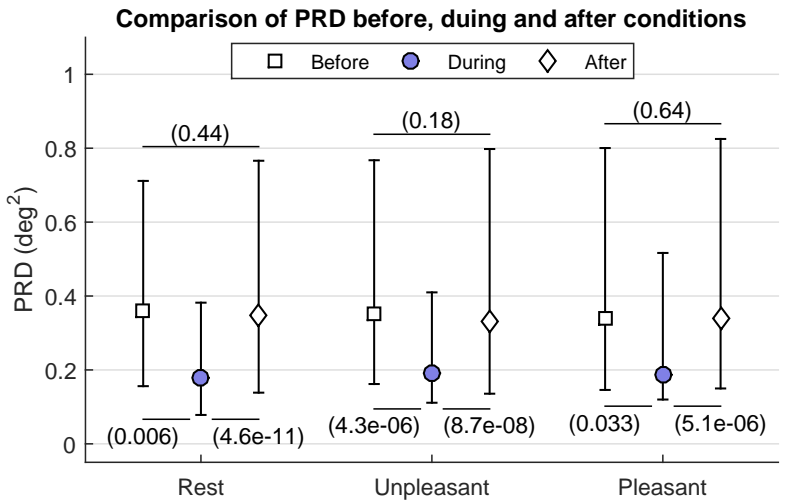

Figure 4. Differences between PRD before, during and after each condition.

\subsection{Music-induced emotions}

Four patients were discarded from the study due to low quality signal, bringing the total number of patients to 71 . The time-course of the baseline corrected PRD was similar during all conditions (Fig. 2): it showed a gradual decrease with respect to the $20 \mathrm{~s}$ interval preceding the condition which lasted about $30 \mathrm{~s}$, and a second phase where the PRD was stable. The analysis of the mean PRD within the interval from $30 \mathrm{~s}$ to $60 \mathrm{~s}$ after the onset of the condition (see $T_{\mathrm{m}}$ in Fig. 2), shows that during both pleasant and unpleasant conditions the PRD was higher as compared to silence (Fig. 3). These differences were small but consistent across participants. Furthermore, the mean PRD was slightly higher when listening to pleasant music than unpleasant acoustic stimuli (Fig. 3, bottom panel). However, these differences were no longer significant after baseline correction (Fig. 3, upper panel). Interestingly, a highly significant difference was found between the mean PRD measured during (see $T_{\mathrm{m}}$ in Fig. 2) and before (see $T_{0}$ in Fig. 2) or after each condition, during which the participants were executing the self-assessment task (Fig. 4). These differ- 
ences were independent of the type of condition and the valence of the acoustic stimuli (Fig. 4). No difference was found between the PRD measured during self-evaluation tasks preceding or following different conditions.

\section{Discussion}

This study investigated 71 young healthy subjects to assess the effect of music-induced emotions on the PRD, an index quantifying low-frequency ventricular repolarization dynamics and associated with sympathetic modulation of the ventricular activity [3]. The main results are: (1) PRD were higher during listening to both pleasant and unpleasant music than during silence; (2) There was a trend for the PRD to be higher during listening to pleasant than unpleasant music, which was however only observed without baseline correction. This may indicate the existence of a (weak) interaction between the valence of music-induced emotions and sympathetic ventricular response; (3) The PRD significantly increased during the $20 \mathrm{~s}$ interval inbetween conditions, during which each participant executed a self-assessment task and waited for the next excerpt. Both cognitive tasks and expectation of an event are associated with sympathetic activation, which may explain the increase observed in PRD. The simulation study shows that the algorithm implemented in this work can accurately track PRD changes with a temporal resolution of approximately $10 \mathrm{~s}$, which was sufficient to describe the dynamics observed in this study. It is worth noting that in its original formulation [3], PRD were calculated using the Scalogram instead of Cohen's class time-frequency distributions, which have been shown to provide a better timefrequency resolution [10].

Future studies may investigate the effect of music-induced emotions on ventricular repolarization dynamics and sympathetic modulation by means of other ECG parameters $[9,12,13]$.

\section{References}

[1] Koelsch S, Jancke L. Music and the heart. European Heart Journal 2015;36(44):3043-3048. ISSN 15229645.

[2] Orini M, Bailón R, Enk R, Koelsch S, Mainardi L, Laguna P. A method for continuously assessing the autonomic response to music-induced emotions through HRV analysis. Medical and Biological Engineering and Computing 2010; 48(5):423-433. ISSN 01400118.

[3] Rizas KD, Nieminen T, Barthel P, Zürn CS, Kähönen M, Viik J, Lehtimäki T, Nikus K, Eick C, Greiner TO, Wendel HP, Seizer P, Schreieck J, Gawaz M, Schmidt G, Bauer A. Sympathetic activityassociated periodic repolarization dynamics predict mortality following myocardial infarction. Journal of Clinical Investigation 2014;124(4):1770-1780. ISSN 0021-9738.
[4] Rizas KD, McNitt S, Hamm W, Massberg S, Kääb S, Zareba W, Couderc JP, Bauer A. Prediction of sudden and non-sudden cardiac death in post-infarction patients with reduced left ventricular ejection fraction by periodic repolarization dynamics: MADIT-II substudy. European heart journal 2017;294:1240-1247. ISSN 1522-9645.

[5] Hanson B, Child N, Van Duijvenboden S, Orini M, Chen Z, Coronel R, Rinaldi CA, Gill JS, Gill JS, Taggart P. Oscillatory behavior of ventricular action potential duration in heart failure patients at respiratory rate and low frequency. Frontiers in physiology 2014;5:414. ISSN 1664-042X.

[6] Pueyo E, Orini M, Rodríguez JF, Taggart P. Interactive effect of beta-adrenergic stimulation and mechanical stretch on low-frequency oscillations of ventricular action potential duration in humans. Journal of Molecular and Cellular Cardiology 2016;97:93-105. ISSN 00222828.

[7] Krabs RU, Enk R, Teich N, Koelsch S. Autonomic effects of music in health and Crohn's disease: The impact of isochronicity, emotional valence, and tempo. PLoS ONE 2015;10(5):e0126224. ISSN 19326203.

[8] Orini M, Hanson B, Monasterio V, Martínez JP, Hayward M, Taggart P, Lambiase P. Comparative evaluation of methodologies for T-wave alternans mapping in electrograms. IEEE Transactions on Biomedical Engineering 2014;61(2):308-316. ISSN 00189294.

[9] Orini M, Taggart P, Srinivasan N, Hayward M, Lambiase PD. Interactions between activation and repolarization restitution properties in the intact human heart: In-vivo whole-heart data and mathematical description. PLoS ONE 2016;11(9):e0161765. ISSN 19326203.

[10] Orini M, Bailon R, Mainardi LT, Laguna P, Flandrin P. Characterization of Dynamic Interactions Between Cardiovascular Signals by Time-Frequency Coherence. IEEE Transactions on Biomedical Engineering 2012;59(3):663673. ISSN 0018-9294.

[11] Orini M, Bailón R, Laguna P, Mainardi LLT, Barbieri R, Bailon R, Laguna P, Mainardi LLT, Barbieri R. A multivariate time-frequency method to characterize the influence of respiration over heart period and arterial pressure. EURASIP Journal on Advances in Signal Processing 2012; 2012(1):214. ISSN 1687-6180.

[12] Laguna P, Martinez Cortes JP, Pueyo E. Techniques for Ventricular Repolarization Instability Assessment From the ECG. Proceedings of the IEEE 2016;104(2):392-415. ISSN 0018-9219.

[13] Ramirez J, Orini M, Tucker JD, Pueyo E, Laguna P. Variability of Ventricular Repolarization Dispersion Quantified by Time-Warping the Morphology of the T-Waves. IEEE Transactions on Biomedical Engineering 2017;64(7):16191630. ISSN 0018-9294.

Address for correspondence:

Name: Michele Orini

Gower Street, London, UK

m.orini@ucl.ac.uk 